\title{
Support vector machine based alzheimer's disease diagnosis using synchrony features
}

\author{
Nilesh Kulkarni \\ Department of Electronics \& Telecommunication, Shrimati Kashibai Navale College of Engineering, India
}

\begin{tabular}{l} 
Article Info \\
\hline Article history: \\
Received Dec 10, 2018 \\
Revised Dec 30, 2019 \\
Accepted Jan 12, 2020 \\
\hline
\end{tabular}

\section{Keywords:}

Alzheimer's disease

Dementia

Electroencephalogram

Machine learning

Support vector machine

\begin{abstract}
Previous research work has highlighted that neuro-signals of Alzheimer's disease patients are least complex and have low synchronization as compared to that of healthy and normal subjects. The changes in EEG signals of Alzheimer's subjects start at early stage but are not clinically observed and detected. To detect these abnormalities, three synchrony measures and wavelet-based features have been computed and studied on experimental database. After computing these synchrony measures and wavelet features, it is observed that Phase Synchrony and Coherence based features are able to distinguish between Alzheimer's disease patients and healthy subjects. Support Vector Machine classifier is used for classification giving 94\% accuracy on experimental database used. Combining, these synchrony features and other such relevant features can yield a reliable system for diagnosing the Alzheimer's disease.
\end{abstract}

This is an open access article under the CC BY-SA license.

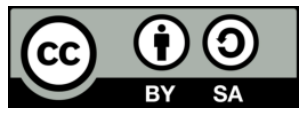

\section{Corresponding Author:}

Nilesh Kulkarni,

Department of Electronics \& Telecommunication,

Shrimati Kashibai Navale College of Engineering,

Gangadhar S Valsepatil Rd, Ambegaon BK, Pune, Maharashtra 411041, India.

Email: nileshkulkarni992@gmail.com

\section{INTRODUCTION}

Alzheimer's disease (AD) is one of the neurodegenerative diseases, characterized by progressive impairment that gradually destroys brain cells and other cognitive functions. Basically, it progressively leads to total dependency. It is most common neurodegenerative disorder particularly in western countries such as United States (US), Canada and many more. Dementia ranks as third most expensive disease and sixth leading cause of death over the world. Approximately, 50-60\% of patients with dementia and Mild Cognitive impairment (MCI) over 65 years of age progresses towards Alzheimer every year [1,2]. Therefore, diagnosis $\&$ effective treatment of patients in early stage are critical and important issues in Alzheimer's research. Alzheimer's disease is basically characterized by wide spread neuronal cell loss, neurofibrillary tangles \& senile plaques in the various brain regions such as hippocampus, entorhinal cortex, neocortex and much more. As number of individuals with Alzheimer's disease is increasing and about to increase in future, diagnosis and effective treatment of disease in early stage are important research challenges. To search for a computational technique for early diagnosis of patients who are progressing towards Alzheimer's, but do no exhibit any clinical symptom of AD during medical tests is an important challenge. Despite of this, an early diagnosis screening method must be inexpensive, in order to allow screening of elderly patient. EEG is one of the most promising tools in such a case.

Neuroimaging techniques play significant role in Alzheimer's diagnosis but they posses several drawbacks as discussed in [3]. Imaging modalities including Magnetic Resonance Imaging (MRI), Computed Tomography (CT), Single Photon Emission Computed Tomography (SPECT) and many more are 
specifically used for diagnosis of various diseases. But, the use of these techniques is also reported in literature. Although these tools are used for Alzheimer's diagnosis, EEG technique is specifically emphasized in current research for Alzheimer's diagnosis. It is most well known and established technique for measuring electric activity of the brain which is generated by billions of neurons of the cerebal cortex. The bioelectric cells are non-invasively examined by series of electrodes placed over patients head as per international 10/20 standards. EEG signal is simply a multivariate signal transmitted over different channels with variation of different sampling frequencies depending on each application [4, 5]. This tool takes more time as it generates more data which needs to be analyzed. EEG signals are key diagnostic tools not only for neurologists, but also for clinicians and doctors. Today, EEG recordings are also used in many Brain Computer Interface (BCI) applications [4]. Several research findings have identified the potential of EEG for diagnosing dementia and Alzheimer's disease in recent years. Since, EEG recording systems are relatively cheap, inexpensive as well as portable. In upcoming years, they can be majorly used for identification of several neuro-degenerative diseases such as Alzheimer's disease, Epilepsy, Huntington disease and many more. Several dynamical changes related to normal ageing can be easily observed using EEG signals [4].

Paper is organized in following manner: Materials and Methods are listed in section 2. Detailed methodology and experimentation is explored in section 3. Classification results and performance metrics are explored in section 4 and Conclusion is summarized in section 5.

\section{MATERIALS \& METHODS}

\subsection{Participants information}

Dataset incorporated in present study consists of electroencephalogram (EEG) recorded in two groups which were aged from 65 to 80 years: SUB1, 50 healthy subjects and SUB2, 50 Alzheimer disease patients which were diagnosed as per NINCDS-ADRDA criteria. Alzheimer's disease patients were grouped as having moderate to severe symptoms. Diagnosis of patients was made by experienced neurologists and expert clinicians on the basis of MMSE and CDR scores. Alzheimer's patients scored below 26 on CDR scale. Dementia and Alzheimer's disease patients were also tested using Single photon emission computed tomography (SPECT) technique for about 14 to 15 months as functional tests such as MMSE and CDR are not sufficient to detect Alzheimer's disease. Another criteria used for detecting Alzheimer's disease was estimating patients functional and cognitive decline for period of last 10 to 15 months based on talk with informants and expert neuroclinicians. Approval was taken from Ethical committee of the hospital and participants relatives related to EEG data and its analysis. All patients were also checked for various diseases such as sugar intake, kidney problems and many more as they can also result into dementia.

\subsection{Recording \& acquisition of EEG data}

EEG signals were recorded and analyzed using 12-bit resolution, $200 \mathrm{~Hz}$ sampling rate EEG machine of RMS manufacturing. Impedance of machine was kept below 10Mohms and the electrodes were placed according to the International 10-20 systems, recommended by American EEG society. Biauricular referential electrodes were also included since they are also important for recording. EEG data was recorded for 15 minutes per patient. Patients were in relaxing mode with eyes closed. EEG signal artifacts were removed by skilled neurophysiologists and clinicians such as blinking effect, drowsiness, muscle artifacts and artifacts generated by machine during recording process. An epoch of 20 seconds was extracted from each EEG signal from left frontal (F3), right frontal (F4), central (C3-C4), Parietal (P3-P4) and temporal (T3) channels for further analysis by visual inspection.

For epoch analysis, 512-point hamming FFT algorithm was used for filtering of EEG data. The window used was of $2.5 \mathrm{sec}$ duration with $90 \%$ overlap between successive windows. Infinite impulse response LPF was used for filtering of EEG data with a $50 \mathrm{~Hz}$ cut-off frequency. The power grid interference was removed using of low pass filter.

\section{METHODOLOGY}

Due to non-stationary characteristic of EEG signal; analyzing EEG signal is not only tedious but also time consuming and difficult. Since, brain waves can be represented in time as well as frequency domain; EEG signals are explored and analyzed in time, frequency and time-frequency domain [5, 6]. MATLAB (2015 version) platform is enabled for executing the algorithms proposed in present research work. In this section, we discuss the preprocessing and feature extraction process in detail. 


\subsection{Block diagran of the proposed system}

Alzheimer's Disease diagnosis system works in different steps, i) Filtering and noise removal of raw EEG signa called Preprocessing, ii) Extraction and selection of prominent features from EEG data, and iii) Classification of EEG data into different subjects. A raw EEG signal was filtered using suitable Bandpass filter to remove unwanted information content from signal which gets added during recording process. In present work, a bandpass filter of third order with appropriate gain designed in between 0.5 to $30 \mathrm{~Hz}$ was used for filtering. In feature extraction stage, synchrony based different features are computed and a training feature matrix was computed as an input to classifier. Block diagram of the system as shown in Figure 1.

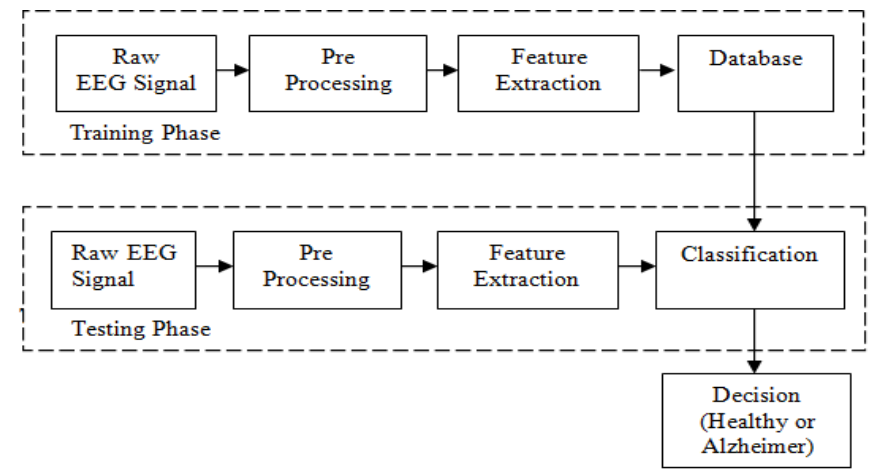

Figure 1. Block diagram of the system

\subsection{Feature extraction using synchrony based features}

In the present research study, synchrony based feqatures were studies and analyzed. Brain Synchrony resembles simultaneous appearance of rhythmic distinct patterns over different regions of head; bilaterally or unilaterally. It is observed that statistical independence appears to be significantly less in case of Alzheimer's patients around different regions of brain [5-7]. To observe this loss of synchrony in brain, synchrony measures such as Magnitude Square Coherence (MSC) and Phase Synchrony which comparatively provide better results in terms of classification accuracy are analyzed. Let us discuss these features in more detail. Magnitude Squared Coherence (MSC). Coherence refers to measurement of synchrony between two independent signals (Let us say x and y) and it can be mathematically defined as,

$$
C_{x y}=\frac{\left|P_{x y}(f)\right|^{2}}{\left|P_{x x}(f)\right| P_{y y}(f) \mid}
$$

Where, $\mathrm{f}$ is frequency in hertz, $\mathrm{P}_{\mathrm{xy}}$ indicates cross spectral density of signal $\mathrm{x}$ and signal $\mathrm{y} . \mathrm{P}_{\mathrm{xx}}$ and $\mathrm{P}_{\mathrm{yy}}$ represents the power spectral densities of $\mathrm{x}$ and y respectively. For each pair of channels namely F3, F4, C3, C4 and O1, O2; coherence features were computed and averaged over the five sub-bands. Coherence highlights the synchronicity of activation in various brain regions. EEG coherence helps to access functional connectivity between various channels over occipital regions of brain. In total, we have computed total (6 channels $* 4$ frequency bands $=24$ features) for 50 subjects consisting of healthy as well as Alzheimer affected in training mode. So, it consists of total $50 * 24=1200$ features.

Phase Synchrony indicates interdependence of signals $\mathrm{x}$ and y among two phases $\emptyset_{x}$ and $\emptyset_{y}$. Even though the amplitudes of signals $\mathrm{x}$ and $\mathrm{y}$ are kept statistically dependent, the instantaneous phases can be strongly synchronized. It is given by following formula [8]:

$$
\emptyset_{m, n}=m \emptyset_{x}(t)-n \emptyset_{y}(t)=\text { constant }
$$

Where co-efficients $\mathrm{m}$ and $\mathrm{n}$ represents integers depicting frequency locking ratio and $\emptyset_{m, n}$ is its relative or phase difference. For computing synchronization between two phases, it is important for us to know the phase of two signals at instantaneous value. It can be analyzed by using Hilbert transform which is given as:

$$
z(t)=x(t)+\tilde{x}(t)
$$


In above equation, $z(t)$ is complex term with is $x(t)$ real term and $\tilde{x}(t)$ represents its Hilbert transform. Hilbert Transform is computed as,

$$
\tilde{x}(t)=\frac{1}{\pi} P V \int_{-\infty}^{\infty} \frac{x(t)}{t-\tau} d t
$$

In above equation, PV represents Cauchy Principle value. Instantaneous phase $\emptyset_{x}(t)$ and $\emptyset_{y}(t)$ for signals $\mathrm{x}$ and $\mathrm{y}$ are calculated as:

$$
\emptyset(t)=\arctan \left(\frac{\tilde{x}(t)}{x(t)}\right)
$$

In present study, synchrony based features were computed on each pair of channel of two opposite regions for all frequency bands and then averaged. For example, phase synchrony feature were measured on typical channels of right and left temporal regions (say (F3-T3), (T3-T4)) and so on. In present study, phase synchrony feature was computed for frontal, temporal, parietal and central electrode of EEG signal. Accordingly, 150 such features were computed and out of it 100 features were selected as per Wilcoxon tests on our dataset [8].

\section{MACHINE LEARNING AND CLASSIFICATION TECHNQUES}

Machine Learning is a subset of Artificial Intelligence in which the data optimization is carried using the past experience to predict future result or classify the target input $[9,10]$. This data optimization can be done using different alogrithms as discussed in literature. For classifying the target input data into two or more classes, different machine learning algorithms can be used. This machine learning algorithms are classified into three learning mechanism namely: supervised, unsupervised and reinforcement learning. In present research work, Support Vector Machine (SVM), a semi-supervised machine learning algorithm was used for classification purpose. For classification purpose, $70 \%$ data consisting of both subjects was incorporated for training purpose and rest of 30\% data was kept for testing purpose. Based on observed results, confusion matrix was created and different performances metric were calculated to measure the efficiency of the classifier. Figure. 2 represents the Semi-supervised machine learning flow.

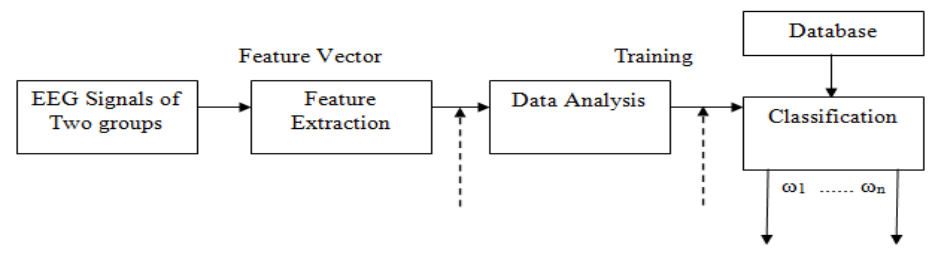

Figure 2. Statistical recognition flow for supervised classification

\subsection{Support vector machine (SVM) classifier}

Classifiers in machine learning are normally formulated, designed and adopted by using statistical learning. They can be used for classification as well as regression purpose. SVM is semi-supervised machine learning algorithm which is very effective in classifying data of high dimension. It is also robust and easy to implement using Python, MATLAB and any other tool. An optical hyperplane separates the data belonging to two classes using appropriate kernel in case of non-linear, multi-dimensional data. In present work, RBF kernel was used and algorithm was implemented using MATLAB programming. Leave-One-Subject-Out (LOSO) cross-validation technique was used for classification. The advantage of using this technique is that it eliminates the problem of over-fitting during data separation and also gurantees the generality of classififer to unseen or unlabelled data.

\subsection{Performance analysis of the EEG data}

Different synchrony-based EEG features such as Magnitude Squared Coherence (MSC), Phase Synchrony and Cross - correlation were computed, analyzed and measured in appropriate manner on EEG data. For selecting training data, computed features were selected from any of the four selected EEG electrodes. Individual features were also computed and tested using SVM classfier based on different performance metrics such as accuracy. The obtained results are shown in Table.1 Based on database available for computation, $50 \%$ of the data was trained \& remaining $50 \%$ data was left out for testing 
purpose. Based on the individual features computed \& SVM classifier used, different performance metrics were obtained based on following terminologies $[9,10]$.

$$
\begin{aligned}
& \text { Accuracy }=\frac{T P+T N}{T P+F N+F P+F N} \\
& \text { Sensitivity }=\frac{T P}{T P+F N} \\
& \text { Specificity }=\frac{T N}{F P+T N}
\end{aligned}
$$

Where, TP stands for True Positive (Alzheimer's disease patients correctly classified), TN stands for True Negative (healthy individuals correctly classified), FP stands for False Positives (healthy individuals misclassified), FN stands for False Negative (Alzheimer's disease patients misclassified).

Table 1. Performance measure on each individual feature

\begin{tabular}{cc}
\hline Features & Accuracy (in \%) \\
\hline Magnitude Squared Coherence & 88 \\
Phase Synchrony & 90 \\
Combination of Both features & 94 \\
\hline
\end{tabular}

\section{CONCLUSION \& RESEARCH CHALLENGES}

This paper presents a method to diagnose Alzheimer's disease by using different Synchrony based features. The aim of the present research work was to improve the diagnostic accuracy for Alzheimer's diagnosis. The features used for differentiating between healthy and Alzheimer's disease subjects are not novel but significant improvements can be observed on used database. In present study, each individual feature was used for classification and all synchrony features were also separately was classification purpose. SVM classifier was used for separating both subjects yielding better results. Spectral analysis also provides better results in analyzing EEG signals. In [11-14], authors have already concluded that significance of plagues and neurofibrillary tangles in cortex regions as well as lower volume of hippocampus slows the signal in case of Alzheimer's disease patients. PET as well as MRI scans was also studied along with MMSE and CDR score since only these are not sufficient for Alzheimer's diagnosis. Previous literature summarizes the investigation of synchrony-based features for Alzheimer's diagnosis. It can be concluded that coherencebased features depict significant effects on signals of Alzheimer's disease patients in resting conditions and high frequency band ranging from 13 to $30 \mathrm{~Hz}$. The synchrony-based features were computed individually and classified by use of semi-supervised classifier; Support vector machine (SVM) giving better classification accuracy for distinguishing the subjects between two groups.

From the features computed as discussed in section 3, it is observed that lower dysfunctional connectivity is observed in central, parietal and occipital brain regions. A lower synchronization in these areas for alpha and beta band identifies disturbance occurred in perception and cognitive disorder. Thus, the observations computed in present research work are in agreement with several previous studies which reports on decrease of neuronal synchrony in Alzheimer's disease patients as observed on EEG signals. This loss of synchrony appears due to addition of non - consistent background activity and presence of neurofibrillary tangles in hippocampus area. Combination of all synchrony-based features provides better accuracy as compared to individual feature. Future work of present research work involves analysis of each EEG signal in time - frequency domain and to conclude whether they cary any relevant information for better detection by using advanced signal processing algorithms.

It is to conclude that when we combine all features together, classification rate as well as diagnostic accuracy obtained is more and it provides comparatively better results in terms of accuracy. Future work in this study involves making analysis of each frequency bands in depth to observe whether they carry any other significant information for better diagnosis by means of various signal processing algorithms and features extraction techniques. The present research work explores a significant methodology for Alzheimer's disease diagnosis and further research using some more of these features can report remarkable achievements in this field.

\section{ACKNOWLEDGEMENTS}

Auhtors would like to thank entire staff of Smt. Kashibai Navale Medical College \& General Hospital for providing necessary EEG database for carrying research work. Authors would also like to thank annoymous reviewers for improving the quality of paper.

Support vector machine based alzheimer's disease diagnosis using synchrony features (Nilesh Kulkarni) 


\section{REFERENCES}

[1] Mattson M., "Pathways towards and away from alzheimer's disease," Nature, vol. 430, pp. 631-639, 2004.

[2] Meek P.D., McKeithan K., and Shumock G.T., "Economics considerations of alzheimer's diseasell," Pharmacotherapy, vol. 18, pp. 68-73, March-April 1998.

[3] Jing Wan, Zhilin Zhang, "Identifying the neuroanatomical basis of cognitive impairment in alzheimer's disease by correlation \& non-linearity-aware sparse bayesian learning," IEEE Trans. on Med. Imaging, vol.33, no.7, pp. 1475-1487, July 2014.

[4] N. N. Kulkarni, V. K. Bairagi, "Electroencephalogram based diagnosis of alzheimer disease," in Proc. IEEE Conference on Intelligent Systems and Control, pp. 1-5, 2015.

[5] Jaeseung Jeong, "EEG dynamics in patients with Alzheimer's disease," Artificial Intelligence in Medicine, vol. 115, no. 7, pp. 1490-1505, July 2004.

[6] Justin Dauwels, et al, "Slowing and loss of complexity in alzheimer's EEG: two sides of the same coin?," Hindawi Access of Research, International Journal of Alzheimer's Disease, vol. 2011.

[7] Raymundo Cassani, et all, "The effects of automated artifact removal algorithms on electroencephalography-based alzheimer's disease diagnosis," Frontiers in Aging Neuroscience, vol. 6, no. 55, pp. 1-13, Mar 2014.

[8] Dhiya Al-Jumeily, Shamaila Iram, Francois-Benois Vialatte, Paul Fergus, and Abir Hussain, “A novel method of early diagnosis of alzheimer's disease based on EEG signals," The Scientific World Journal, vol. 2015, Article ID 931387, 11 pages, 2015. doi:10.1155/2015/931387

[9] Andrea Rueda, Fabio A. Gonzalez, "Extracting salient brain patterns for imaging-based classification of neurodegenerative diseases," IEEE Trans. Med. Imaging, vol. 33, no.6, pp. 1262-1274, June 2014.

[10] Suresh, M., Ravikumar, M., "Dimensionality reduction and classification of color features data using SVM and KNN," International Journal of Image Processing and Visual Communication, vol. 1, no. 4, pp. 2319-1724, February 2013.

[11] Nilesh Kulkarni, "Use of complexity based features in diagnosis of mild Alzheimer disease using EEG signals," International Journal of Information Technology, vol. 10, no. 1, pp. 59-64, 2018.

[12] N. N. Kulkarni, V. K. Bairagi, "Extracting Salient features for EEG based Diagnosis of Alzheimer's disease Using Support Vector Machine classifier," IETE Journal of Research, Taylor and Francis, vol. 63, no. 1, pp. 11-22, March 2017.

[13] N. Houmani et al., "Diagnosis of Alzheimer's disease with Electroencephalography in a differential framework," PLoS One, vol. 13, no. 3, 2018.

[14] Justin Dauwels, Francois-Benoit Vialatte, and Andrzej Cichocki, “On the early diagnosis of alzheimer's disease from EEG Signals: A mini-review,” Advances in cognitive Neurodynamics, pp. 709-716, 2010.

\section{BIOGRAPHIES OF AUTHORS}

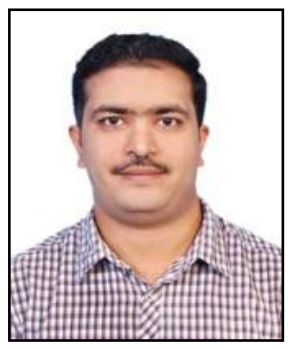

Nilesh Neelkanth Kulkarni was born in Pune, India, in 1992. He received B.E. degree in Electronics engineering from the University of Pune, Pune, India, in 2013, and the M.E. degree in Electronics \& Telecommunication engineering from Savitribai Phule Pune University (Formerly, University of Pune) in 2015, respectively. In 2016, he joined the Department of Electronics Engineering; All India Shri Shivaji Memorial Society's Institute of Information Technology, as a Visiting faculty and then continued as Assistant Professor in Electronics Engineering department. Presently, he is working as Assistant Professor in Smt. Kashibai Navale College of Engineering, Pune. His current research interests include Biomedical Engineering, Biomedical Signal \& Image Processing, Machine Learning and Pattern Recognition. He is a member of the Institution of Engineers (India); The Institution of Electronics and Telecommunication Engineers (IETE). He is a Life Member of IEI (India), IETE and Biomedical Engineering Society of India. He has filed one patent at national level. He received "Best Innovative Project Award" for his research project presented at WIECON - 2016 sponsored by IEEE, Pune Section. He is recipient of Prof. M. N. Saha Memorial Award, awarded by IETE, India. He has published 16 research papers at International level. Presently, he is working on Biomedical Circuits and System applications. 